\title{
TRA PRINCIPIO DI EGUAGLIANZA E RESPONSABILITÀ: DIVERSI ITINERARI DI TUTELA DEL MINORE
}

\author{
BETWEEN THE PRINCIPLE OF EQUALITY AND RESPONSIBILITY: DIFFERENT \\ ROUTES FOR THE PROTECTION OF THE CHILD
}

\section{Virginia Zambrano}

Professore ordinariodi Diritto Privato Comparatonell' Universitàdi Salerno. È stata Docente di Diritto Anglo-americano e Diritto Delle Comunità Europee Per La Seconda Università di Napoli; èdottorediricerca e si è specializzata presso La Faculté Internationale de Droit Comparé in Strasburgo. Componente

Del Consiglio Direttivodella Scuoladi Specializzazi one per Le Professioni Legalidell' Universitàdi Salerno. È responsabile di progetti di ricerca finanziatidal Ministerodell' Università e della Ricerca. Aderisceall'Associazione Italiana di DirittoComparato. Collabora con l'Instituto De Desarrollo

Y Análisis Del Derecho De Familia En España (IDADFE).

E-mail: vpozzetti@gmail.com

Convidada

RIASSUNTO: Scopo di questa ricerca è quello di evidenziare come il minore sia innanzitutto "persona", rispetto alla quale occorre declinare i principi di uguaglianza e responsabilità. La metodologia utilizzata è di tipo deduttivo. Lo studio si avvale dei contributi della giurisprudenza e della dottrina sia italiana che straniera.Infine, la ricerca è qualitativa. Si intende rilevare come la tutela del minore dipenda da una serie di profiliche si collegano ad una etica sociale della legalità e dei diritti umani. Il diritto ad una famiglia, alla casa di abitazione, il diritto a conoscere le proprie origini sono alcuni degli aspetti sui quali si è inteso riflettere, per dimostrare su cosa poggia la protezione del minore. Questi è un essere in formazione, sia dal punto di vista fisico che psichico, e ha bisogno di essere tutelato in quanto persona. Non si tratta di vedere solo cosa stabilisce la norma: per rendere efettiva la tutela occorrono adeguate politiche pubbliche in grado di garantire l'applicazione dei principi di uguaglianza e responsabilità, nonché etici. Sia nel campo teorico che nell'attuazione delle politiche pubbliche, occorre assicurare lo sviluppo della personalità del minore.

Parole-chiave: status filiationis; principi di uguaglianza e responsabilità; personalità del minore.

SOMMARIO: Introduzione - 1. Filiazione e minore - 2. Status filiationis e nascita di una relazione complessa -3 . Il primo segreto della storia del minore -4 . Diversi itinerari di lettura: crisi della coppia e casa familiare - 5. A proposito di falsi miti: il minore protagonista e la informed doctrine formula. Conclusione. Bibliografia. 


\section{INTRODUZIONE}

Quando si discorre di minore non è concepibile avere pregiudizi siano essi fondati su discriminazioni razziali o condizionati dallo status filiationis. Tanto meno sono ammissibili discriminazioni legate al fatto che il minore è figlio di una coppia omosessuale o eteroaffettiva. Il minore è un essere in formazione che deve essere rispettato nella sua interezza, perché solo in questo modo si può costruire una società più giusta ed egualitaria.

Lo scopo di questa ricerca èanalizzare i principi di uguaglianza e responsabilità riguardo ai diritti del minore, non solo in termini di protezione, ma anche avuto riguardo alla sua protezione come "persona". In quest'ottica si è inteso guardare alla dottrina e alla giurisprudenza per verificare il livello di protezione assicurato al minore rispetto ad alcuni profili, come il diritto di abitazione e il diritto a conoscere le proprie origini biologiche.

Il problema è: come assicurare al minore, qualunque sia il suo status filiationis, il rispetto della dignità umana e dei diritti in modo che possa essere realizzata la sua formazione integrale, senza offuscarne lo sviluppo della personalità?

La giustificazione per questa analisirisiede nel fato che, nonostante le astratte previsioni normative, ancora non si è approdati ad unadeguato livello di protezione dei diritti del minore. Il diritto a vivere nella casa di famiglia (quando i genitori si separano), il diritto di avere una famiglia, il diritto del figlio adottivo o nato da tecniche di fecondazione assistita a conoscere le proprie origini stentano a trovare attuazione; così come stenta a trovare riconoscimento il diritto del minore ad esprimere il proprio consenso al trattamento medico, a dispetto di una dottrina che pure riconosce al minore la capacità di discernimento.

Dal punto di vista metodologico, la ricerca ha inteso analizzare la legislazione, la dottrina e la giurisprudenza - sia italiana che straniera - per poi approdare ad una conclusione logica. La ricerca è di natura qualitativa, in quanto non cercha di identificare dati percentuali o quantitativi.

\section{FILIAZIONE E MINORE}

Ogni indaginesulla famiglia e sulle relazioni familiari, pur partendoda basi rigorosamente giuridiche, nonpuò essere avulsa dal contesto sociale e culturale di riferimento ${ }^{1}$. La comparazione insegna che il diritto ha bisogno delle altre scienze sociali; che il diritto non condiziona lo sviluppo sociale, ma lo riflette; che dal confronto con le scelte degli altri ordinamenti europei, si possono ricavare elementi significativi, delineare trend, idonei a tracciare un cammino comune, sebbene percorso a differenti velocità ${ }^{2}$.La premessa è tanto più significativaquando si discorre di tutela del minore e, più in generale, di tutela dell'infanzia.Si incontra qui una generale convergenza degli ordinamenti che fa emergere, almeno sul piano dei principi, la compiuta metabolizzazione dell'idea che il minore va protetto e, al tempo stesso, rispettato come persona.

Nel continuumdell'esistenza umana l'infanzia - e sul punto v'è concordia - rappresenta null'altro che una fase nella vita della persona; momento nel quale occorre creare le condizioni che rendano possibile un armonico sviluppo della personalità. Il minore, dunque, non è né un adulto in miniatura né un oggetto da possedere e tanto meno quel soggetto "muto" cui pure la parola infanzia rinvia.

La complessità epistemologica del concetto di persona investe allo stesso modo l'adulto e il minoreche è persona in formazione e, dunque, soggetto da tutelare con i suoi bisogni, desideri, debolezze, volontà, interessi. Soggetto che va capito, rispettato e curato nella sua specificità. Il

\footnotetext{
${ }^{1}$ È l'insegnamento di G. AUTORINO STANZIONE, Introduzione, in Il diritto di famiglia nella dottrina e nella giurisprudenza. Trattato teorico-pratico, Torino, $2^{\mathrm{a}}$ ed., 2011, I, p. XV ss.

${ }^{2}$ Per un analitico excursus di detta evoluzione cfr. M. SESTA, Diritto di famiglia, Padova, 2005, $1 \mathrm{ss.}$
} 
fondamento della tutela del minore affondacosì le sue radici autentiche nella tutela dei diritti umani "la cui collocazione sta nell'etica sociale della giuridicità, ed è suscettibile di specificazioni diverse in funzione della specificità delle singole culture sociali, nella cornice dei tratti essenziali che identificano ciascuna figura" ". La dimensione propria della tutela del minore è l'universo della persona, dove i suoi diritti ricevono riconoscimento generale per il tramite di un processo di aggregazione culturale e politica che parte dalla comuneaccettazione e da una comune visione dei diritti umani.

Lastessa nozione di interesse del minorenon si sottrae a tale dinamica. In discussione, quando si discorre di interesse del minore, non è allora la definizione dei suoi contenuti che andrebbe incontro alle stesse difficoltà qualificatorie che caratterizzano i diritti umani. Il rischio di queste letture, pur quando condotte con lo sguardo rivolto alla giurisprudenza, risiede nel privilegiare un approccio meramente descrittivo che non rende giustizia al significato e alla funzione dell'espressione in esame.

Ogni tentativo condotto in tale direzione, restituisce solo risultati parziali prodotto di visioni, interpretazioni che si alimentano delle diverse lenti con cui si guarda al minore (come all'uomo) e dei diversi contesti riferimento; ricerca, dunque,inutile, come lo è ogni tentativo di cogliere la specificità del contingente e la singolarità dell'esistenza ${ }^{4}$.D'altro canto quello di interesse del minore, più che alla complessità dell'esperienza umana, deve la propria indefinibilità al suo offrirsi alla riflessione del giurista in quanto principio che proclama un valore, un fine e che, quindi, come tutti i principi che veicolano un valore non definisce "precisamente in che modo esso dovrà essere realizzato"s.

Nella pragmatica della comunicazione giuridica, il richiamo all'interesse del minore serve allora per veicolare un messaggio ben preciso. L'obiettivo è, per un verso,coinvolgere il legislatore in un'opera di costruzione della norma in grado di trasferire valori e principi ritenuti prevalenti e, per l'altro, offrire all'interprete le chiavi di lettura appropriate per dare attuazione a quel valore. Ma quello di interesse del minore è - come si diceva - concetto complesso che, oltre ad ispirare il legislatore, opera sia da un punto di vista sostanziale che processuale.

Il consenso sulla necessità di predisporre adeguati strumenti di tutela del minore si fonda quindi sulla esigenza di evitare che l'età possa rappresentare un ostacolo allo sviluppo della persona o, peggio, risolversi in abuso o essere fonte di differenze. In questa dimensione valoriale trovano spazio gli artt. 3, 9 e 12 della Convenzione delle Nazioni Unite sui diritti dell'infanzia del 1989nonché la Convenzione di Strasburgo del 1996. Ed in questa direzione ancora muove l'art. 24 della Carta di Nizza, non a caso,posto nel Capo III dedicato all'uguaglianza.

Ma se è vero che punto di partenza ed insieme di arrivo nel dibattito sulla tutela del minore sono i principi generali collegati ai diritti fondamentali e alla tutela della persona e,

\footnotetext{
${ }^{3}$ A. FALZEA, Nel cinquantenario della convenzione europea sui diritti dell'uomo, in Riv.dir.civ., 2000, p. 696.

${ }^{4} \mathrm{~J}$. CARBONNIER, Les notions à contenu variable dans le droitfrançais de la famille, inC. Perelman et R. Vander Elst (dir.), Les notions à contenu variable en droit, éd. Bruxelles. E. Bruylant, 1984, p. 99. J. GOLDSTEIN, FREUD, ANNA e SOLNIT, ALBERT, In The Best Interests Of The Child, New York Free Press 1986, passim. Per una attenta e completa definizione dell'interesse del minore si rinvia a P. STANZIONE, Interessi del minore $e$ < <statuto>> dei suoi diritti fondamentali, in Fam. e dir., 1994, p. 351 ss.; G. AUTORINO, Diritto di famiglia, Torino, 2003, p. 195. In proposito, altresì, G. CASABURI, Nota a Cass, civ. 20 gennaio 2006, n. 1202, in Foro it., 2006, 5, c. 1410 ss. nonché F. RUSCELLO, La tutela dei figli nel nuovo <〈affidamento condiviso〉>, in Familia, 2006, passim; A. SCALISI, Famiglia e diritti del minore, in Fam. pers. e succ., 2006, 10, p. 817 ss.; C. M. BIANCA, La nuova disciplina in materia di separazione dei genitori e affidamento condiviso: prime riflessioni, in Dir. fam. $e$ pers., 2006, XXXV, 2, p. 678; G. SALITO, L'affidamento condiviso dei figli nella crisi della famiglia, in Diritto di Famiglia. Trattato teorico-pratico, dir. da G. Autorino Stanzione, Torino, 2007, p. 231 ss. Sul punto sia consentito, altresì, il rinvio a V. ZAMBRANO, Interesse del minore ed affidamento coniugato. Esperienze europee a confronto, in Dir. fam. e pers., 2000, 3-4, p. 1385.

${ }^{5}$ G. ZACCARIA, Precomprensione, princìpi e diritti nel pensiero di Josef Esser. Un confronto con Ronald Dworkin, in "Ragion pratica", 1998, 6, p. 145; G. PINO, Principi e argomentazione giuridica, in "Ars Interpretandi. Annuario di ermeneutica giuridica", 2009, p. 144.
}

Revista de Direito Brasileira | Florianópolis, SC | v. 23 | n. 9 | p. 389-409 | Mai./Ago. 2019 
dunque, eguaglianza, non discriminazione, diritto a vivere nella famiglia di origine e ad intrattenere rapporti familiari è anche vero che alla armonizzazione sembra frapporsi ladiversità socio-culturale e tecnico-giuridica dei contesti di riferimento. Ed, infatti, allorchè dal piano delle astratte valutazioni si passa a verificare in concreto le scelte operate dai diversi ordinamenti, ci si avvede che - a dispetto delle formule declamatorie utilizzate- tutt'altro che omogeneo è il livello di tutela del minore.

A volte si tratta di incoerenze significative che conferiscono instabilità al dibattito sulla "convergenza" degli ordinamenti e sulla ampiezza del livello ditutela riconosciuto al minore ${ }^{6}$, altre volte le differenze sono più sfumate e si colgono al livello della regola di dettaglio dovute, come sono,al diverso modo in cui all'interno dell'ordinamento dialogano tra loro i formanti. Status filiationis, diritto del minore a conoscere le sue origini, capacità si prestano in siffatto contesto a rappresentare le coordinate di una riflessione, attraverso la quale si vuole verificare il livello di protezione assicurato al minore.

\section{STATUS FILIATIONIS E NASCITA DI UNA RELAZIONE COMPLESSA}

La messa in discussione dell'unicità del modello familiare ${ }^{7}$ segue l'insegnamento di Carbonnier: "à chacun sa famille, à chacun son droit". La dissociazione fra matrimonio e filiazione ricombina l'universo familiare. E se l'adozione testimonia della separazione fra sessualità, riproduzione e filiazione, le tecniche di riproduzione assistita frammentano il processo del concepimento o chiamano in causa più corpi femminili.

La possibilità per le coppie omosessuali di accedere alle tecniche di riproduzione assistita o all'adozione e al riconoscimento giuridico del legame che li unisce al minore contribuisce alla dissociazione fra procreazione e filiazione e apre una nuova era nella quale la differenza di sesso non è condizione imprescindibile per la nascita di un rapporto di filiazione. In ragione della complessità delle relazioni interpersonali, adozione omoparentale, surroga di maternità, fecondazione eterologa si intrecciano dando vita a conflitti giurisprudenziali particolarmente significativi, come quelli concernenti "i figli di due madri" ${ }^{\circ}$ " $i$ figli di due padri" ${ }^{\text {Nel }}$ processo di combinazione e ricombinazione delle tessere del mosaico familiare ciò che appare chiaro è il progressivo smarrimento di confini cui si assiste quando si mette mano alla costruzione giuridica del rapporto di filiazione. Alla tradizionale componente "genetica" o "procreativa", si affiancano

${ }^{6}$ H. FULCHIRON, Existe-t-il un modèle familial européen?,Defrénois 2005, p. 1461.

${ }^{7}$ V. SCALISI, La "famiglia" e le "famiglie" (il diritto di famiglia a dieci anni dalla riforma), in Scritti catanzaresi in

onore di Angelo Falzea, Napoli, 1987, p.431 ss.; ID., "Famiglia" e "famiglie" in Europa, relazione svolta al convegno

Persona e comunità familiare, 1982-2012, in www.comparazionedirittocivile.it; V. CARBONE, Crisi della famiglia e principio di solidarietà, relazione svolta al convegno Persona e comunità familiare, 1982-2012, ibidem; C. MAZZÙ, Nuovi modellidi status nel diritto di famiglia, ibidem; F.D. BUSNELLI, La famiglia e l'arcipelago familiare, in Riv. dir. civ., 2002, I, p. 509 ss.

${ }^{8}$ Sulla trascrizione dell'atto di nascita del figlio nato in Spagna, in seguito al concepimento avvenuto tramite donazione dell'ovulo dalla moglie della donna (e fecondazione con gamete di donatore anonimo) che lo aveva partorito:

Cass.civ., 30 settembre 2016 n. 19599, in http://dirittocivilecontemporaneo.com/wp-content/uploads/2016/10/Cass.30-settembre-2016-n.-19599.

${ }^{9} \mathrm{La}$ questione riguarda la trascrizione dell'atto di nascita dei gemelli nati negli Stati Uniti tramite surroga di maternità, indicati alla nascita come figli anche del suo consorte secondo la legge del luogo di nascita dei minori: App. Trento, ord. 2 febbraio 2017, in http://www.articolo29.it/wpcontent/uploads/2017/02/Ordinanza . Sul punto, altresì App. Milano, decr. 28 ottobre 2016, http://www.articolo29.it/wp-content/uploads/2017/01/Corte-appMilanotrascrizione-nascita-gemelli. Dove in discussione era la trascrizione dell'atto di nascita dei gemelli nati dalla fecondazione di due distinti ovuli, con il seme di due aspiranti padri, riconosciuti come tali secondo la legge californiana.

Revista de Direito Brasileira | Florianópolis, SC | v. 23 | n. 9 | p. 389-409 | Mai./Ago. 2019 
infatti quella biologica, quella fondata sulla legge (adozione) ovvero quella sociale, dando vita ad un intreccio di situazioni generative di uno status unico, quello di figlio. Sì che lacontrapposizione fra legame giuridico, legame sociale e legame biologico sempre più si delinea come prodotto di una costruzione culturale che registra la "liquidità" delle relazioni familiari ${ }^{10}$.

Il linguaggio giuridico appunta il cambiamento di prospettiva e supera la pluralità deglistatusfiliationis, tentando difar coincidere qualificazione giuridica del rapporto e dimensione relazionale. Nell'intreccio fra verità e responsabilità si muovono così le riforme europee in tema di filiazione.

La riforma francese del $2005^{11}$ coglie questo aspetto e, preferendo discorrere di paternità e di maternità, prende distanza dalle condizioni necessarie alla costruzione giuridica del vincolo per guardare piuttosto al momento della responsabilità.Su questo crinale si è incamminato (seguendo le esperienze europee) anche il legislatore italiano con la 1. 219/2012 e con il dlgs. 154/2013 (emanato per adeguare il codice civile ai principi introdotti con la legge 129/2012). La legge italiana è il risultato ultimo di un processo di modernizzazione delle relazioni familiari che si era avviato nel 1975 con la legge di riforma del diritto di famiglia. Ed in vero, sebbene già nel ' 75 si fossero create le premesse per dare attuazione alla previsione di cui all'art. 30 della Cost., i segni della diversità di trattamento fra figli legittimi e naturali si avvertivano nella a) conservazione di due distinti stati di filiazione (legittima e naturale); b) inammissibilità dell'acquisizione dello stato di figlio da parte di chi è stato generato in seguito ad incesto; c) inesistenza di parentela tra il figlio ed i parenti dell'altro genitore (art. 74 c.c.); d) attribuzione del cognome; e) disciplina sulla introduzione del figlio naturale nella famiglia legittima del genitore ai fini della convivenza; f) facoltà di commutazione in danaro della quota ereditaria sugli immobili spettante ai figli naturali; g) mancata previsione della possibilità di succedere fra fratelli naturali (art. 565 c.c.). Questo rigoroso panorama normativo era però stato progressivamente travolto da una serie di interventi da parte della Corte costituzionale che, pur sottolineando l'irragionevolezza di qualsiasi distinzione fra filiazione dentro o fuori del matrimonio, non aveva perso l'occasione per ribadire comunque la priorità del modello famiglia fondata sul matrimonio ${ }^{12}$. Il timore di stravolgere un

${ }^{10} \mathrm{D}$. BORILLO, La vérité biologique contre l'homoparentalité: le statut du beauparentou le «PaCS de la filiation», in Droit et société 2009/2, n. 72, p. 259-271.

${ }^{11}$ Ordonnance n. 2005-759 su cui J. MASSIP, Le nouveau droit de la filiation, in Rép. Defrenois, 2006, pp. 6 ss., 91 ss., 209 ss.; T. GARÉ, L'ordonnance portant réforme de la filiation, in JCP, G 2006, I, p. 144; L. MAUGERVIELPEAU, La volonté et la filiation au regard du droit nouveau. (A propos de l'Ordonnance n. 2005-759 du 4julliet 2005 portant réforme de la filiation), in Pet. Aff., 27 juin 2007, 128, p. 3 ss.

12 Nonostante l'importanza riconosciuta alla famiglia legittima, i giudici non esitano a dichiarare l'illegittimità costituzionale - per contrasto con gli artt. 3 e 30, comma terzo, cost. - dell'art. 565 c. c., nella parte in cui esclude dalla categoria dei chiamati alla successione legittima, in mancanza di altri successibili, e prima dello Stato, i fratelli e le sorelle naturali riconosciuti o dichiarati (Corte cost. 4 luglio 1979, n. 55, in Foro it., 1979, I, 1941; Corte cost. 12 aprile 1990, n. 184, in Nuove leggi civ. comm., 1990, 1454, con nota di M. Costanza; Corte cost. 7 novembre 1994 , n. 377, in Rass. dir. civ., 1995, I, 84, con nota di F. Prosperi; Corte cost. 23 novembre 2000, in Giust. civ., 2001, 591, con nota di M.C. Bianca). In dottrina F.D. BUSNELLI, Sui criteri di determinazione della disciplina normativa della famiglia di fatto, in Famiglia di fatto. Atti del convegno di Pontremoli, Pontremoli, 1977, p. 133; F. SANTOROPASSARELLI, Parentela naturale, famiglia e successione, in Riv. trim. dir. proc. civ., 1981, p. 27; in argomento cfr. M. Costanza, in Comm. dir. it. fam., Cian, Oppo, Trabucchi, V, Padova, 1992, sub art. 566, p. 99. La difficoltà di assicurare piena parità di trattamento alla filiazione naturale era comunque da mettere in relazione alla mancanza di un intervento del legislatore. Ma a fronte dei forti cambiamenti sociali, dicono i giudici, "appare sempre meno plausibile che i fratelli e sorelle naturali del de cuius restino esclusi dalla successione ab intestato a vantaggio anche di lontani parenti legittimi fino al sesto grado" (sent. 377/1994). Il rammarico, che investe l'assenza del legislatore, non impedisce ai giudici di continuare a riflettere su quelle disposizioni del codice civile che fanno emergere una disparità di trattamento. Così si dichiara l'incostituzionalità dell'art. 803 , comma 1 , cc. nella parte in cui prevede che - in caso di sopravvenienza di un figlio naturale - la donazione possa essere revocata solo se il riconoscimento del figlio sia intervenuto entro due anni dalla donazione. Scompare progressivamente dalle motivazioni della Corte ogni richiamo alla "clausola di compatibilità". La famiglia legittima sembra conservare la sua centralità solo in tema di costruzione del legame di parentela, come si desume da decisioni nelle quali si nega rilevanza alla c.d. "parentela naturale", affermando che l'equiparazione fra filiazione legittima e filiazione naturale richiesta dall'art. 30, co. 3,

Revista de Direito Brasileira | Florianópolis, SC | v. 23 | n. 9 | p. 389-409 | Mai./Ago. 2019 
dettato costituzionale che - pur riconoscendo l'eguaglianza morale e giuridica dei coniugi, salvo i limiti disposti a garanzia della unità familiare, art. 29 Cost.- proclama che la famiglia è fondata sul matrimonio, lasciava ai giudici i margini interpretativi di cui all'art. 2 Cost. Il cambio di prospettiva si compie nel momento in cui passa a privilegiare la prima parte dell'art. 29 Cost., laddove si discorre di famiglia come "società naturale" a indicare, non solo la sua priorità rispetto allo Stato, ma anche l'apertura allo sviluppo delle dinamiche sociali ${ }^{13}$.

Il progressivo riconoscimento del fatto che "l'ingresso di figli naturali in un rapporto coniugale e in una vita familiare legittima di per sé non è una violazione di diritti ma un incerto del mestiere di vivere" segna l'affievolimento definitivo del primato assegnato alla famiglia legittima ${ }^{14}$.

In Italia, sono la dottrina e la giurisprudenza, dunque, a creare in Italia le condizioni per quell'intervento del legislatore che rende il figlioinfine "protagonista della vicenda familiare" La legge 129/2012 modifica la stessa nozione di famiglia legale, la quale non appare più necessariamente fondata sul matrimonio e attribuisce rilievo all'interesse prioritario del minore. La importanza che assume la modifica dell'art. 74 c.c. è solo un esempio del rilievo attribuito alla nozione di parentela, come relazione fondata sulla generazione e non più sul matrimonio. Corollario di questa modifica è stata la immediata riscrittura delle norme sulla successione legittima e necessaria ${ }^{16}$.

Ma a cambiare, nella prospettiva dell'interesse del minore è la stessa natura della relazione che si instaura fra questi e il genitore. Il riferimento alla responsabilità genitoriale, che si sostituisce all'espressione potestà genitoriale, muove nella direzione del riconoscimento ai genitori di poteri che sono funzionali alla crescita della personalità del figlioe al suo benessere.In Francia (loi 2002-305) all'idea dell'exercice conjoint de l'autorité parentalesi sostituisce un concetto di "coparentalitè" che prescinde del tutto da quella coabitazione che, in caso di separazione o divorzio, fa invece dire al legislatore spagnolo, a norma dell'art. 156 Código Civil, che la potestà "se ejercerá por aquel con quien el hijo conviva". In Germania è invece un giudice costituzionale, particolarmente attento, che si preoccupa disegnalare l'incostituzionalità della normaladdove non prevede l'esercizio congiunto della potestà e a stimolare l'adeguamento del§ 1626a BGB nel senso di ritenere cheder elterlichen Sorge beiden Eltern gemeinsamhaben ${ }^{17}$.Salvo poi, più di recente, osservare che l'affidamento congiunto funziona soltanto se a dialogare sono entrambi i genitori giacchè, in caso contrario, è proprio la valutazione dell'interesse del minorea preferire l'affidamento esclusivo ${ }^{18}$.

In generale, però, aspirazione egalitaria fra coiniugi e principio di eguaglianza dei figli (senza distinguere a seconda del tipo di rapporto che hanno fra loro i genitori) influenzano le riforme relative all'esercizio dell'autorità genitoriale. Filiazione biologica, adozione,

cost. riguarda soltanto il rapporto che si instaura tra il genitore e il figlio (cfr. sent. n. 363 del 1988; 184 del 1990; 377 del 1994; 532 del 2000). In dottrina, sul punto, E. LAMARQUE, Art. 30, in Commentario alla Costituzione, a cura di R. Bifulco-A. Celotto-M. Olivetti,Torino, 2006, vol. I, p. 634 ss.).

${ }^{13}$ R. BIN, La famiglia: alla radice di unossimoro, in Studium juris, 2000, p. 1068. Le "nuove famiglie" e la parificazione degli status di filiazione ad opera della L. 219/2012. Atti dei Convegni di Milano, 7 marzo 2014 Bolzano, 21 marzo 2014 - Salerno, 13 giugno 2014, in I Quaderni della Fondazione Italiana del Notariato, n. $3 / 2014$.

${ }^{14}$ Così i giudici in Corte Cost., 494/2002.

${ }^{15}$ M.G. STANZIONE, Il diritto alla genitorialità e alle relazioni familiari, in www.comparazionedirittocivile.it, p. 5.

${ }^{16}$ G. FERRANDO, Stato unico di figlio e varietà dei modelli familiari, in Fam.dir., 2015, p. 952. Osserva l'A. come a seguito della Riforma i figli a) hanno diritti sulla successione legittima dei parenti entro il $6^{\circ}$ grado; b) ai genitori e agli ascendenti (senza ulteriori aggettivazioni) è riconosciuta una quota di riserva sulla successione del figlio o nipote; c) la rappresentazione in linea collaterale opera a favore dei discendenti dei fratelli (prima solo dei fratelli legittimi).

${ }^{17}$ BVerfG, 3 nov. 1982, BVerfGE 61.358.

18 OLG Stuttgart 24 agosto 2016 in Aktenzeichen 17 UF 40/16. Più in generale, sulla necessità di approdare ad una valutazione concreta di ciò che corrisponde all'interesse del minore, anche tenedo conto delle sue aspirazioni, cfr., BVerfG, FamRZ, 2008, s. 1737 ff. E, in dottrina, Staudinger/Coester (2015) § 1666 Rn. 66. 
procreazione assistita, maternità surrogata fondano la genitorialità su di un rapporto che,legandosi a responsabilità, supera anche la dimensione biologica ${ }^{19}$.Ilrichiamo alla unicità dello status guarda, in questo senso, alla dimensione sociale della famiglia come luogo dove si sviluppano una serie di relazioni interpersonali che assumono significato proprio nell'interesse del minore.

Ed allora, modalità di costituzione del vincolo, ricerca della verità come ricerca della identità, e assunzione di responsabilità designano i poli attorno ai quali si costruisce "un sistema filiazione" che vede nella tutela del minore il suo punto di riferimento. Fra le pieghe di un dettato normativo che riconosce anche le unioni civili e, dunque, non solo la convivenza, ma pure le unioni omoaffettive, $(76 / 2016)^{20}$ si sviluppa così in Italia una giurisprudenza che alla "liquidità" delle relazioni familiari risponde avvalendosi di schemi i quali - come nel caso della possibilità di adozione del figlio del partner (cd.stepchild adoption) - arrivano a "stravolgere" una norma, l'art. 44, comma 1, lett. d), 1. 183/1984 che, in realtà, disegna un procedimento adottivo speciale solo per i coniugi.L'interesse del minore alla stabilità della relazione affettiva supera simili irragionevoli preclusioni normative e si apre anche alla possibilità di ammettere l'adozione del figlio del partner della coppia omosessuale.

I giudici, nonostante la legge discorra unicamente di "coniuge" non hanno cioè esitato ad estendere la fattispecie anche al convivente dello stesso sesso ${ }^{21}$, specie allorchè tra l'aspirante adottante e l'adottando si sia instaurato uno stabile rapporto, corrispondente all'esercizio della responsabilità genitoriale ${ }^{22}$.

Ed è proprio in questa apertura alla realtà sociale -e sul presupposto del riconoscimento del diritto del minore a mantenere le relazioni affettive con i componenti del contesto familiare chetrova spazio altresì il riconoscimentodella "genitorialità sociale". L'obiettivo che persegue la giurisprudenzaitaliana (più attenta ai cambiamenti sociali di quanto non lo sia il legislatore) è quello di consentire al figlio,che sia passato a vivere con la nuova famiglia del proprio genitore, di mantenere un rapporto continuativo anche con il genitore sociale, purse successivamente la coppia è andata in crisi ${ }^{23}$.

Emblematico, in proposito, il ragionamento che fanno i giudici in Corte Cost. 225/2016. In discussione, nella caso di specie, era il diritto del minore ad avere rapporti significativi non solo con ascendenti e parenti (come chiaramente disposto dall'art. 337ter c.c.) ma anche con il convivente della madre. I giudici, senza spingersi a dichiarare l'illegittimità costituzionale dell'art. 337ter c.c., ritengono che il diritto del figlio a "conservare rapporti significativi" con persone diverse dai genitori debba essere inteso in senso lato. L'allontanamento della convivente della madre (pur in assenza di qualsiasi vincolo giuridico con il figlio della donna) è ritenuto condotta "pregiudizievole" per il figlio e, dunque, sanzionabile ex art. 333 c.c., norma che dà la facoltà al giudice di assumere, a tutela del minore, "i provvedimenti convenienti" necessari. Per il tramite di una interpretazione sistematica delle norme del codice civile alla luce dei principi costituzionali, in cui alla parola "genitore" è attribuito un significato che prescinde dall'esistenza di un vicolo giuridico o di sangue, i giudici evitano di affrontare la questione della legittimità costituzionale dell'art. 337ter c.c.

\footnotetext{
${ }^{19} \mathrm{~J}$. LONG, Il diritto italiano della famiglia alla prova delle fonti internazionali, Milano, 2006, 68 ss.

${ }^{20}$ In GU Serie Generale n.118 del 21 maggio 2016.

${ }^{21}$ Cass. Civ. 27 settembre 2013 n. 22292 CED Cassazione, 2013 e, da ultimo, sul fatto che la relazione omoaffettiva sottostante l'adozione non può essere considerata contrastante, "in re ipsa", con l'interesse della minore, in quanto una tale valutazione negativa, fondata esclusivamente sull'orientamento sessuale della coppia, si risolverebbe in un'inammissibile discriminazione, Cass. civ., 22 giugno 2016, n. 12962, in Foro it., 2016.

${ }^{22}$ E. ANTONIO EMILIOZZI, L'adozione da parte di partners di unioni civili, in Riv.trim.dir.proc.civ., fasc.2, 1, 2018, pag. 799; V. CARBONE, Riconosciute le unionicivili tra persone dello stesso sesso e le convivenze di fatto, in Fam. e dir., 2016, pp. 856-857; G. FERRANDO, La disciplina dell'atto. Gli effetti: diritti e doveri, in Fam. e dir., 2016, p. 894; Trib.min. Roma, 22 ottobre 2015, in Foro it., 2016, I, c. 339 ss.

${ }^{23}$ Cfr. Corte cost., 5 ottobre 2016, n. 225.
}

Revista de Direito Brasileira | Florianópolis, SC | v. 23 | n. 9 | p. 389-409 | Mai./Ago. 2019 
Nel caso dell'adozione alla coppia omosessuale, così come per le situazioni di genitorialità sociale, ciò che si intende assicurare al minore è la continuità delle relazioni familiari, nella consapevolezza che la rete di contesto del minore è fondamentale per lo sviluppo della persona.

Un obiettivo, questo,che trova conferma tanto nella previsione dell'imprescrittibilità dell'azione di disconoscimento della paternità per il solo figlio (art. 244 , comma $4^{\circ}$, c.c.), quanto nelle disposizioni di cui alla 1.19 ottobre 2015, n. 173 che, proprio in nome della continuità affettiva, permettealla coppia affidatariadi poter adottare il minore che loro hanno in affido ${ }^{24}$.

\section{IL PRIMO SEGRETO DELLA STORIA DEL MINORE}

Non v'è dubbio che filiazione, identità, interesse del minore siano aspetti strettamente connessi. Osserva Paul Ricoeur, «répondre à la question “qui?”, c'est raconter une histoire» ${ }^{25}$. L'identità dunque arriva a definire la persona, disegna le coordinate di una individualità che si riflette anche nel modo in cui socialmente è percepita la persona.

L'identità è il diritto ad essere se stessi e affermare la unicità della propria persona. Diritto fondamentale che si scompone in una serie di aspetti fisici, psicologici, biologici, sociali. A sottolineare la complessità della questione un giurista italiano, Stefano Rodotà, sottolineava come l'identità fosse paragonabile ad un vero e proprio "pozzo"sul quale "troppi giuristi evitano ancora di spingersi" 26 .

La difficoltà di indagine è nella molteplicità dei fattori che concorrono a definire l'identità di una persona,cosa che rende impossibile delinerane il concetto o offrire una spiegazione della sua funzione ${ }^{27}$. Di diritto del fanciullo a "preservare la propria identità" discorrono gli art. 7 e 8 della Convenzione di New York, mentre 1'art. 30 della Convenzione dell'Aja ${ }^{28}$ impone agli Stati firmatari non solo l'obbligo di "conservare con cura le informazioni in loro possesso sull'origine del minore" ma anche di "assicurare l'accesso del minore o del suo rappresentante legale a tali informazioni, nella misura in cui sia consentito dalle leggi dello Stato".

Ora, a dispetto di una apparente sovrapponibilità di contentuti fra diritto all'identità e diritto a conoscere le proprie origini, il dato che emerge dall'analisi delle diverse esperienze europee è rappresentato dalla necessità di tener separati i due profili. L'autonomia fra accertamento del vincolo di filiazione e azioni condotte per conoscere la realtà biologica si riflette in una giurisprudenza che sembra essere consapevole del fatto che quello all'identità è un diritto più ampio, che racchiude la percezione sociale del sé e non si risolve nella mera componente genetica ${ }^{29}$.

La complessità del diritto all'identità è nel suo presentare, accanto ad una componente statica, una veste dinamica che attinge alla dimensione sociale della persona. E così, se da un

\footnotetext{
${ }^{24}$ P. MOROZZO DELLA ROCCA, Sull'adozione da parte degli affidatari dopo la L. n. 173/2015, in Fam. e dir., 2017, p. 602 ss.

${ }^{25}$ I. Théry, La distinction de sexe. Une nouvelle approche de l'égalité, 2007, Paris Odile Jacob.

${ }^{26}$ V. ZENO ZENCOVICH, voce Identità personale, in Digesto delle discipline privatistiche, IX, Torino, 1993, $\mathrm{p}$. 294.

${ }^{27}$ Di identità declinata in una dimensione diacronica, come possibilità di rimanere se stessi nel tempo, discorre D. GUTTMANN, Le sentiment d'identité. Étude de droit des personnes et de la famille, Paris, 2000, passim.

${ }^{28} \mathrm{La}$ Convenzione sulla protezione dei minori e sulla cooperazione in materia di adozione internazionale è stata firmata all'Aja il 29 maggio 1993 ed è ratificata in Italia con 1. 31 dicembre 1998, n. 476.

${ }^{29}$ In questosenso i giudicidella Suprema Corte de Justicia della Provincia di Buenos Aires in un sentenza del 26 marzo 2013 osservano" el concepto de identidad filiatoria non esnecessariamente el correlato del dato puramente genético determinado por la procreación; va mucho más allá; por eso una cosa es tener el derecho a conocer ese dato, y otra, muy distinta, la pretensión de tener vínculos jurídicos, fundados en ese dato génetico". Cfr., A.M. CHECHILE, El derecho humano de acceder a la verdad biológica sin generar vínculos jurídicos, in Derecho de Familia. Revista Interdisciplinaria de Doctrina y Jurisprudencia, 2006, vol.III, p. 162 ss.
}

Revista de Direito Brasileira | Florianópolis, SC | v. 23 | n. 9 | p. 389-409 | Mai./Ago. 2019 
punto di vista "statico",il richiamo all'identità si cogliein una serie di dati quali nome, religione, cittadinanza, nascita etc. che servono ad individuare la persona, da un punto di vista dinamico,essa,si riferisce a quella complessa rete di relazioni sociali che rende l'uomo partecipe di una comunità dialogica ${ }^{30}$.Osserva Fichte che ognumo di noi diventa uomo perché invitato ad essere uomo sottolineando come il punto di partenza antropologico sia proprio la relazione che consente alla persona di determinare se stesso $^{31}$.In questa accezione dinamica, allora, l'identità non si combina solo con l'elemento biologico, genetico, ma sottende una componente culturaleche investe anche la costruzione del legame familiare.La filiazione, d'altro canto, si osserva “n'est pas seulement un droit de la vérité. C'est aussi un droit de la vie, de l'intérêt de l'enfant, de la paix des familles, des affections, des sentiments moraux, du temps qui passe" 32 .

Il diritto a conoscere le origini si afferma, per contro, come diritto fondamentale autonomo rispetto all'identità ed attinge la sua ragion d'essere nella necessità di conoscere le radici della propria esistenza e- come nel caso dell'adozione - di un vissuto che ha comunque generato un contesto relazionale.Certo, anche la nascitacrea relazione.Il diritto a conoscere le originiassume però un rilievo diverso a seconda si discorra di adozione o di altri modi di costituzione del vincolo.Il diritto a conoscere le origini va cioè incontro a delle "variazioni in tema"condizionate dal modo in cui è originato il rapporto.

Nel caso dell'adozione la conoscenza delle origini genetiche si associa - come si diceva con la scoperta di un vissuto che connotail profilo dinamico dell'identità e spiega i maggiori limiti che circondano il diritto a conoscere le origini. Nel secondo - all'opposto - ad occupare le scena è la mera conoscenza dell'identità genetica. In entrambe le situazioni alla esigenza di conoscere si contrappone - ed è tensione che interessa tutti gli ordinamenti -il diritto all'anonimato di chi ha donato il seme, della madre porteur ovvero della madre che ha chiesto di non essere nominata nell'atto di nascita.

L'importanza della componente relazionale è invariabilmente avvertita dai legislatori nel caso dell'adozione. La successiva costruzione giuridica del rapporto adottivo non annulla la "verità naturale", non cancella pregressi vissuti, ma invita la famiglia adottiva a facilitare la conoscenza.

In questa direzione muove, ad esempio, la previsione del nuovo Codice civile argentino(art. 596 del CCYC argentino)e gli artt. 235-49 y 235-509 Codi Civil de Catalunya. Il coinvolgimento della famiglia adottiva si sostanzia nel suo "comprometerse expresamente a hacer conocer su origenes al adoptado" (art. 596 CCYC argentino). Come dire che il genitore adottivo, coinvolto nel processo educativo, ha l'obbligo di agevolare la ricostruzione di un vissuto che può essere funzionale all'armonico sviluppo della personalità dell'adottato. Sebbene sia difficile parlare di un vero e proprio obbligo del genitore di cui sarebbe, peraltro, complesso far valere l'inadempimento, quello che vale la pena rilevare è il tentativo di dare rilievo giuridico ad un obbligo morale. È una strada sulla quale non si incammina il legislatore italiano, il cui intervento di riforma del del $2001^{33}$ si arresta alle soglie di una generica disposizione normativa che tuttavia rimette ai genitori il compito di "informare" l'adottato (art. 24 1. 149/2001). Ma a differenza di quanto accade in Spagna, essi sono lasciati soli nella scelta del quando e delquomodo rendere l'informazione.

Nulla, dunque, che richiami la forza con cui in Spagna l'art. 180 CC ammette "El derecho a conocer los datos sobre sus orígenes biológicos" imponendo, altresì, alle Entidade públicas

\footnotetext{
${ }^{30}$ F. C. SESSAREGO, Derecho a la identidad personal, Buenos Aires, 2012, p. 15 ss.; M. HERRERA, E. LAMM, Sub art. 564, in Tratado de derecho de familia, Según el Código civil y commercial de 2014, t. II, artt. 509-593, Buenos Aires, 2014, p. 558 ss.

${ }^{31}$ J.G.FICHTE, La missione del dotto, La Nuova Italia, Firenze 1939 e 1973, passim.

${ }^{32}$ G. CORNU, Droit civil. La famille, Paris, Montchrestien, 9e éd., 2006; D. BORILLO, La vérité biologique contre l'homoparentalité: le statut du beau-parento u le "PACS de la filiation", in Droit et sociétés, vol. 2, pp. 259271.

${ }^{33}$ L. 28 marzo 2001, n. 149.
}

Revista de Direito Brasileira | Florianópolis, SC | v. 23 | n. 9 | p. 389-409 | Mai./Ago. 2019 
l'obbligo di creare le condizioni che consentano di rendere effettivo il diritto. Vero è che qui, come in Italia, si va alla ricerca del limite cui subordinare 1'esercizio del diritto; del pari vero che il legislatore spagnolo preferisce ricondurlo(a differenza di quanto fa il legislatore italiano che fissa il limite nel compimento del 25 anno di età) alla maggiore età che, presuntivamente, si ritiene segni l'acquisto di una maturità tale da consentire l'accesso alle informazioni sulla famiglia naturale.

Ma se quello dell'età è limitedi "protezione" posto a tutela del minore, la possibilità di cancellare ogni riferimento alla famiglia di origine, procedendo a quella "nueva inscripción de nacimiento"34 di cui all'art. 307 (Decreto de 14 de noviembre de 1958, por el que se aprueba el Reglamento para la aplicación de la Ley del Registro Civil) dà nuovamente spazio al diritto della madre all'anonimato, a conferma di quanto difficile sia rendere effettivo il diritto del minore in presenza di altra posizione giuridica. Tanto, senza considerare che la Ley $N^{\circ}$ 14/2006, ( $\operatorname{artt} .5 .5$ e 21) in tema di tecniche di riproduzione assistita, introduce un principio di segretezza delle informazioni relative al donante la cui identità non può essere rivelata, a meno di esigenze eccezionali.

Nondimeno, che il diritto a conoscere le origini si affermi come un diritto autonomo da far valere con una azione indipendente dalle azioni di stato, si ricava dall'art. 235-49.2 Codi Civil de Catalunya a norma del quale 'L'adoptat, a partir de l'assoliment de la majoria d'edat o de l'emancipació, pot exercir les accions que condueixin a esbrinar la identitat dels seus progenitors biològics, la qual cosa no afecta la filiació adoptiva".E, su queste basi, un legislatore attento non esita addirittura ad imporre alla pubblica amministrazione la predisposizione di strutture e di centri di assistenza in grado di favorire l'acquisizione di queste informazioni.

D'altro canto, nel silenzio del diritto comune, a destare l'attenzione sono le soluzioni adottate proprio da taluni Derechos Forales i quali hanno scelto di inserire il principio in epigrafe nelle leggi che si occupano di mediazione familiare, prevedendosi qui un procedimento speciale il quale consente di soddisfare, in maniera pacifica e consensuale, il diritto di ogni adottato alle proprie origini biologiche ${ }^{35}$.

Diversamente è a dirsi nei casi di surrogazione di maternità ovvero di fecondazione assistita.

L'approccio seguito dagli ordinamenti è condizionato dalla presenza in Europa di due diversi modelli giuridici di attribuzione della maternità. Ad un modello fondato su una attribuzione, per dir così, obbligatoria della maternità (Germania), si contrappone un modello volontaristico nel quale (come in Francia o in Italia, Spagna, art. 113 Còdigo civil) il rigore del principio mater semper certaè attenuato da un legislatore che subordina la costruzione del rapporto di filiazione alla manifestazione di volontà della madre e alla possibilità di non essere nominata nell'atto di nascita. La necessità di soddisfare esigenze di certezza fa sì che, in presenza di tecniche di fecondazione assistita, ovvero di maternità surrogata, alcun risalto sia attribuito alla volontà procreazionale,e il rapporto di filiazionesi costruisca in relazione a colei che ha generato (cfr. per l'esperienza italiana l'art. 269, comma 3, c.c.; art. 8, 1. 40/2004).

In quei sistemi giuridici - Francia, Italia -che conoscono la regola secondo cui la dichiarazione di volontà è essenziale per la nascita dello status filiationis, e che consentono alla donna diconservare l'anonimato ${ }^{36}$ il problema centrale è stato quello di scegliere fra il diritto della donna all'oblio e quello del figlio a conoscere le proprie origini. Nella direzione della costruzione

\footnotetext{
${ }^{34}$ Di cui alla Disp. Prim. Instrucción de 15 de febrero de 1999 para la adopción nacional y Resolución-Circular de 31 de octubre de 2005 en materia de adopciones internacionales.

35 J. LEDESMA DEL BUSTO, Mediación familiar en búsqueda de orígens. El encuentro con mi espejo biológico, Madrid, 2012, passim.

${ }^{36}$ In Italia, l'art. 30 comma 1, del d.P.R. n. 396 del 2000 (Regolamento per la revisione e semplificazione dell'ordinamento dello stato civile, a norma dell'articolo 2 comma 12, della 1.15 maggio 1997, n. 127) prevede che "la dichiarazione di nascita è resa da uno dei genitori, da un procuratore speciale, ovvero dal medico o dalla ostetrica o da altra persona che ha assistito al parto, rispettando l'eventuale volontà della madre di non essere nominata".
}

Revista de Direito Brasileira | Florianópolis, SC | v. 23 | n. 9 | p. 389-409 | Mai./Ago. 2019 
del rispetto integrale della identità del minore una grande "partita" è così stata giocata da una giurisprudenza europea che, per un verso,lo ha inquadrato come "diritto della personalità" e, per l'altro, ha sottolineato l'impossibilità di ammettere un diverso rilievo del diritto alle origini a seconda che si discorra di adozione ovvero di fecondazione assistita ${ }^{37}$. In questo modo la giurisprudenza europea ha contribuito alla creazione di un ambiance culturale comune, pur in presenza di profonde differenze fra i diversi ordinamenti.

In Germania, ad esempio,dove "Mutter eines Kindes ist die Frau, die es geboren hat" $\S$ 1591 BGB, la possibilità per la madre di "ihre Identität nicht preisgeben"38 (§ 2 abs.4 Schwangerschaftskonfliktgesetz - SchKG) ha rappresentato un deciso mutamento di prospettiva. La previsione del parto anonimo ${ }^{39}$ disciplinata nel 2013 attraverso un ampio emendamento alla SchKG apread una "vertrauliche Geburt" ( $\$ 25$ Gesetz zum Ausbau der Hilfen für Schwangere und zur Regelung der vertraulichen Geburt) che non fa venir meno la possibilità per il figlio che abbia compiuto i 16 anni di conoscere l'identità della madre ( $\$ 63$ del PersonenstandGesezt (PstG).

Anche qui, nondimeno, l'azione è autonoma rispetto alle azioni di stato, come si desume dal fatto che il ricorso alle prove biologiche non è previsto allo scopo di mettere in discussione il legame giuridico della filiazione $(\S 1598 \mathrm{a} \text { BGB })^{40}$. Come dire che la prevalenza del diritto all'anonimato appare ingiustificata a fronte dell'importanza attribuita ai diritti dell'adottato alla conoscenza delle proprie origini, nonché alla salute (ben potendo il segretoostacolare la conoscenza di eventuali problemi genetici) e che l'accertamento della "relazione naturale" non fa venir meno il significato e la funzione del vincolo giuridico.

Né le cose sono andate diversamente in Italia dove, complice un dettato normativo che escludeva l'accesso alle informazioni se l'adottato non fosse stato riconosciuto alla nascita dalla madre naturale (art. 28, comma 7, 1. 183/1984), il formante giurisprudenziale ha mostrato una maggiore "resistenza" alle sollecitazioni che venivano dalla CorteEDU.

Nel contrasto fra diritto all'oblio della madre $e$ esigenze di tutela dell'identitàdel minore i giudici, confortati da un tessuto normativo piuttosto univoco (e che vieta la diffusione di dati riservati ${ }^{41}$ ), hannoconsentitol'accesso alle informazioni solo in presenza di quei motivi di

\footnotetext{
${ }^{37}$ Mikulić v. Croatia (1st Section, Judgment of 4 September 2002, Application $N^{\circ}$ 53176/99), § 54; Odièvre v. France (Judgment of 13 February 2003, Application No 42326/98), § 49; Gaskin v. The United Kingdom (Judgment of 7 July 1989, Application $N^{\circ}$ 10454/83), $\S 37$ e 49; Pretty v. the United Kingdom (Judgment of 29 July 2002, Application $\mathrm{N}^{\mathrm{o}}$ 2346/02), § 61; Bensaid v. The United Kingdom (Judgment of 6 May 2001, Application $\mathrm{N}^{\circ}$ 44599/98), § 47; Ebru et Tayfun EnginÇolak c. Turquie (2nd Section, Arrêt de 30 Août 2006, Requête $N^{\circ}$ 60176/00), $\S$ 83. Sul punto, L. BALESTRA, E. BOLONDI, La filiazione nel contesto europeo, in Fam. dir., 2008, p. 309 ss.

${ }_{38}$ Gesetz zur Vermeidung und Bewältigung von Schwangerschaftskonflikten (Schwangerschaftskonfliktgesetz SchKG)

${ }^{39}$ Gesetz zum Ausbau der Hilfen für Schwangere und zur Regelung der vertraulichen Geburt, in $B G B L$, n. 53 del 03.09.2013, in http://www.bmfsfj.de/BMFSFJ/gleichstellung\%2cdid=199564.html e in particolare cfr., Die vertrauliche Geburt. Informationenüber das GesetzzumAusbau der HilfenfürSchwangere und zurRegelung der vertraulichenGeburt: http://www.bmfsfj.de/BMFSFJ/Service/publikationen,did=206324.html.

${ }^{40} \mathrm{Gesetz}$ zur Klärung der Vaterschaft unabhängig vom Anfechtungsverfahren, in BGLB, del 31.03.2008; A. LAMARCA I MARQUE, Autonomía privada e intervención pública en las acciones de filiación. La reforma del $B G B$, in http://www.indret.com/pdf/561_es.pdf.

${ }^{41}$ Cfr. Dlgs. 30 giugno 2003, n. 196 come modificato dal D.lgs n. 101 del 10 agosto 2018. Recita l'art. 93 (Certificato di assistenza al parto) "1. Ai fini della dichiarazione di nascita il certificato di assistenza al parto è sempre sostituito da una semplice attestazione contenente i soli dati richiesti nei registri di nascita. Si osservano, altresì, le disposizioni dell'articolo 109. 2. Il certificato di assistenza al parto o la cartella clinica, ove comprensivi dei dati personali che rendono identificabile la madre che abbia dichiarato di non voler essere nominata avvalendosi della facoltà di cui all'articolo 30, comma 1, del decreto del Presidente della Repubblica 3 novembre 2000, n. 396, possono essere rilasciati in copia integrale a chi vi abbia interesse, in conformità alla legge, decorsi cento anni dalla formazione del documento. 3. Durante il periodo di cui al comma 2 la richiesta di accesso al certificato o alla cartella può essere accolta relativamente ai dati relativi alla madre che abbia dichiarato di non voler essere nominata, osservando le opportune cautele per evitare che quest'ultima sia identificabile".
}

Revista de Direito Brasileira | Florianópolis, SC | v. 23 | n. 9 | p. 389-409 | Mai./Ago. 2019 
eccezionale gravità di cui parla la legge ${ }^{42}$.Il superamento di questa interpretazione restrittiva si compie nel momento in cui i giudici della Corte costituzionale, ragionando sull'impianto normativo del "parto anonimo" (Corte Cost. 278/2013) rilevano che esso non consente al figlio di venire a conoscenza delle proprie origini, sì che "negare, a priori l'autorizzazione all'accesso alle notizie sulle proprie origini, in ragione del fatto che il genitore abbia dichiarato di non voler essere nominato, compromette il diritto all'identità personale dell'adottato" ${ }^{43}$.

Nel 2013, dunque, i giudici costituzionali riflettono sulla legittimità costituzionale del "parto anonimo" e censurano l'assenza di un meccanismo che consenta di verificare che la madre non ha cambiato la propria volontà. In questo modo essi correggono la posizione espressa nel 2005 (Corte cost. 425/2005) allorché,osservandoche la norma di cui all'art. 28 1. 184/1983 "era espressione della ragionevole valutazione comparativa dei diritti inviolabili dei soggetti interessati", non avevano ritenuto di poter far proprie le considerazioni emerse in Odièvre c. Francia $^{44}$. Qui la Corte di Strasburgo aveva riconosciuto che la legge francese(loi n. 2002-93 del 22 gennaio 2002) riusciva a contemperare i due interessi in questione, rafforzando le possibilità per la donna di revocare la sua decisione e permettendo attraverso un organismo ad hoc - il Consiglio nazionale per l'accesso alle origini personali (CNAOP) - di gestire la reversibilità del segreto, condizionandolo all'accordo espresso dalla madre e dal figlio.

E, tuttavia, benchè nel 2013 i giudici costituzionali abbiano chiaramente ribadito che quello all'identità vada costruito come un diritto della personalità (art. 2 Cost.), e che il rispetto della volontà della madre vada subordinato alla verifica della sua attualità, la sentenza non ha risolto una serie di problemi applicativi.

In discussione era la possibilità per i giudici di procedere direttamente a verificare l'attualità della volontà dell'anonimato ${ }^{45}$ ovvero attendere comunque l'intervento del legislatore che definisse le modalità dell'interpello. Confortati da un impianto normativo immutato,cui per lungo tempo si era sovrapposta una interpretazione incline a privilegiare il diritto all'oblio della madre, i giudici italiani sono rimasti preda di una sorta dipath dependence, culturale, sì da sviluppare due orientamenti contrapposti.

L'ultimo atto di questa vicenda - in attesa di un organico intervento del legislatore - è scritto ancora una volta dai giudici, questa volta della Cassazione i quali, chiamati a risolvere il contrasto interpretativo che aveva fatto seguito alla decisione della Corte cost., hanno chiarito che "il diritto del figlio a conoscere le proprie origini costituisce un elemento significativo nel sistema costituzionale di tutela della persona" e che, in quest'ottica, "il figlio può chiedere al giudice di interpellare la madre ai fini della revoca della dichiarazione, a suo tempo fatta, di non volere essere menzionata come madre nell'atto di nascita" ${ }^{46}$. Sulla decisione della Corte Costituzionale

\footnotetext{
${ }^{42}$ Cfr., l'art. 28, comma 7, legge 183/1984 a norma del quale "L'accesso alle informazioni non e' consentito nei confronti della madre che abbia dichiarato alla nascita di non volere essere nominata ai sensi dell'articolo 30, comma 1, del decreto del Presidente della Repubblica 3 novembre 2000, n. 396". Tra le tante sentenze che hanno fatto applicazione rigorosa del principio, Trib. min. Napoli, 24 luglio 1998; Trib. min. Perugia 21 giugno 2002, in Giur. merito, 2003, p. 42; Trib. min. Perugia 19 luglio 1999, in Dir. fam., 1999, p. 1260; in Giur. merito, 2000 , p. 319 In senso contrario, per un caso isolato, Trib. min. Perugia 4 dicembre 2001, in Giust.civ., 2002, p. 417; Trib. min. Salerno 19 luglio 2002, in Giur. merito, 2003, p. 249.

43 Cfr., Con questa argomentazione si è dischiarata l'illegittimità costituzionale dell'art. 28 comma 7 della legge n.184 del 1983 (in materia di adozione) nella parte in cui non prevede la possibilità per il giudice di interpellare la madre che abbia dichiarato di non voler essere nominata ai sensi dell'art. 30, comma 1, del D.P.R. 3 novembre 2000, n. 396: Corte cost. 18 novembre 2013 n. 278, in Nuova giur. civ. comm., 2014, 285 ss. La Corte, peraltro, si era già pronunciata sul tema con la sentenza n. 425 del 2005, ma aveva escluso l'illegittimità costituzionale della previsione che ammetteva l'anonimato: C. Cost., A.O. Cozzi, La Corte costituzionale e il diritto di conoscere le proprie origini in caso di parto anonimo: un bilanciamento diverso da quello della Corte europea dei diritti dell'uomo?, in Giur. cost., 2005, 4602 ss.

${ }^{44}$ CorteEDU, 13 febbraio 2003 ric. n. 42326/98, Odièvre c. Francia, in Familia, 2004, 1109.

${ }^{45}$ Corte di Appello di Salerno, decreto del 7-8 febbraio 2017.

${ }^{46}$ Cass. Civ., Sez. Un., sentenza 25 gennaio 2017 n. 1946 (Pres. Rordorf, est. Giusti).
}

Revista de Direito Brasileira | Florianópolis, SC | v. 23 | n. 9 | p. 389-409 | Mai./Ago. 2019 
(prima) e delle Sezioni Unite della Corte di cassazione (poi) ha, dunque,certamente"pesato" una giurisprudenza europea che già in Godelli v. Italia aveva sottolineato la irragionevole rigidità della normativa italiana, che non assicura un adeguato bilanciamento fra il diritto all'oblio della madre e il diritto a sapere del figlio ${ }^{47}$.

Il legislatore italiano così resta in attesa, incapace di intervenire nel dibattito e di scrivere la parola fine di una storia che, pur fra contrasti, vede coinvoltoun diritto di rango pretorio più attento a dare attuazione alleistanze di tutela del minore e ad adeguarsi alle sollecitazioni che vengono d'oltralpe. Nella stessa linea di ragionamento si collocano i giudici in Cass. civ., 9 novembre 2016 , n. $22838^{48}$. I giudici - non potendo interpellare la madre che si era avvalsa della facoltà di non essere nominata nell'atto di nascita del figlio, poi dato in adozione, in quanto nel frattempo era morta - conformemente a quanto statuito da Corte cost. 278/13, riconoscono il diritto del figlio a conoscere le proprie generalità, sempre che il trattamento dei dati concernenti la identità personale del figlio sia compiuto in maniera lecita "senza cagionare danni, anche non patrimoniali, all'immagine, alla reputazione e ad altri beni di primario rilievo costituzionale di eventuali terzi interessati (discendenti, familiari)" 49 .

\section{DIVERSI ITINERARI DI LETTURA: CRISI DELLA COPPIA E CASA FAMILIARE}

Se, come appare chiaro, l'interesse del minore è la linea che ispira corti e legislatori, complice una sensibilità che si è sviluppata anche grazie all'apporto di carte e documenti internazionali, differente è la lettura che ne viene offerta.

La dichiarazione di astratta adesione al best interest principle che si incontra in tutti gli ordinamenti subisce delle declinazioni differenziate e la concreta attuazione dell'interesse del minore - di là dai discorsi sulla sua non semplice definizione - appare rimessa alla sensibilità dei giudici e alla loro capacità di andare alla ricerca di soluzioni in grado di intercettarne i contenuti.

Un esempio, per più versi emblematico, è offerto dalla questione dell'assegnazione della casa familiare. In Italia, il nucleo della disciplina si rinviene nell'art. 337 sexies, comma 1, c.c. ${ }^{50}$ a norma del quale è il prioritario interesse del figlio a condizionare il godimento della casa familiare. La precedente previsione di cui all'art. 155 , comma 4 , c.c. che attribuiva, di preferenza, il diritto di abitazione al coniuge affidatario si giustificava sulla base di un regime di affidamento mono-genitoriale ${ }^{51}$ che la legge 54/2006 aveva provveduto ad eliminare.Ciò avrebbe dovuto suggerire ai giudici l'opportunità di prendere le distanze da sentieri già percorsi che però solo si giustificavano sotto il vigore della precedente disciplina.

In realtà, proprio sullo sfondo delle innovazioni introdotte dalla 1. 154/2013, si è sviluppata una giurisprudenza, che ha resistito al cambiamento di prospettiva, suggestionata

\footnotetext{
${ }^{47}$ Godelli v. Italia, i giudici osservano come nel perimetro di tutela offerto dall'art. 8 della Cedu, rientri anche il diritto di conoscere le proprie origini, ed ha quindi accusato la normativa italiana di eccessiva rigidità in quanto non permetteva un bilanciamento dei diritti in gioco, riconoscendo una prevalenza ingiustificata al diritto della madre all'anonimato. Per i giudici “70. La Cour note qu'à la différence du système français examiné dans l'arrêt Odièvre, la législation italienne ne tente de ménager aucun équilibre entre les droits et les intérêts concurrents en cause. En l'absence de tout mécanisme destiné à mettre en balance le droit de la requérante à connaître ses origines avec les droits et les intérêts de la mère à maintenir son anonymat, une préférence aveugle est inévitablement donnée à cette dernière". Attualmente in Parlamento sono in discussione due progetti di legge che mirano a modificare la l. 1983/184 (proposte di legge C-2919 del 11.11.2009 e S-1898 del 18.11.2009).

${ }^{48}$ Foro it. 2016, 12, I , c. 3784.

${ }^{49}$ Cass. civ., 9 novembre 2016, in Foro it., 2016, 12, I , c. 3784.

${ }^{50}$ Introdotto dall'art. 55, dlgs. 28 dicembre 2013, n. 154. Ex multis, Cass., 19 giugno 2013, n. 18440, in Dejure online. Su questi aspetti, G. CARAPEZZA FIGLIA, Assegnazione della casa familiare ed interessi in conflitto, in La casa familiare nelle esperienze giuridiche. Quaderni di Diritto delle Successioni e della Famiglia, 4, Napoli, 2016, p. 98 ss.

${ }^{51}$ Articolo abrogato dall'art. 106, d.lg. 28 dicembre 2013, n. 154, con effetto dal 7 febbraio 2014.
}

Revista de Direito Brasileira | Florianópolis, SC | v. 23 | n. 9 | p. 389-409 | Mai./Ago. 2019 
dall'idea che unicamentela garanzia di continuità dell'habitat domestico possa tutelare adeguatamente l'interesse del minore.

Il legislatore, all'art. 337 sexies c.c., nel prevedere che la casa è attribuita "tenendo prioritariamente conto dell'interesse dei figli”, intendeva offrire al giudice la possibilità di valersi di uno strumento duttile di apprezzamento dell'interesse del minore, per un verso, superando l'equazione fra affidamento della prole eassegnazione al genitore affidatario dell'abitazione e, per l'altro, consentendo soluzioni più flessibili nella definizione dei tempi e modi di realizzazione dell'affidamento.Poco o nulla delle intenzioni del legislatore si rinviene fra le pieghe di una giurisprudenza impegnata ad offrire una lettura restrittiva di "casa familiare". Quando discorrono di "casa familiare" i giudici italiani fanno essenzialmente riferimento ad una nozione oggettiva della stessa, sì che casa familiare è solo "quella nella quale si svolgeva la vita della famiglia allorché era unita" ${ }^{52}$. Funzione dell'assegnazione, per i giudici italiani, èquella di assicurare "l'interesse a permanere nell'ambiente domestico in cui i figli sono cresciuti, per mantenere le consuetudini di vita e le relazioni sociali che in esso si radicano (Cass. 6979/2007, 16398/2007, $14553 / 2011,21334 / 2013)^{\prime \prime 53}$. Per cui ben si giustifica un provvedimento di revoca dell'assegnazione della casa familiare allorchè il minore si allontani per ragioni di studio o abiti saltuariamente la casa ${ }^{54}$.

Difetta ai giudici italiani una visione più ampia che permetta di assumere decisioni non ispirate esclusivamente dal timore di comprimere eccessivamente l'interesse del titolare del diritto di proprietà ${ }^{55}$ ma che guardi alla possibilità di individuare una "dimora idonea, intesa come luogo di formazione della personalità del minore"56. La giurisprudenza italiana sembra rimanere così preda di uno stereotipo culturale che nega le potenzialità di cui all'art. 337 sexies, comma 1, c.c. ${ }^{57}$ il quale intendeva appunto evitare ogni automatismo applicativo.

Vieppiù non trova giustificazione siffatta linea interpretativa ove si pensi allo spazio attribuito all'autonomia delle parti nella definizione degli aspetti personali e patrimoniali conseguenti alla crisi del matrimonio(d.1. 12 settembre 2014, n. 132) ${ }^{58}$. Ispirato dalla necessità di degiurisdizionalizzare il contenzioso, il legislatore italiano ha previsto che i coniugi,con l'assistenza dell'avvocato, e salvo la autorizzazione del giudice, possano negoziare i contenuti personali e patrimoniali dell'accordo di separazione (art. 6) e, quindi, anche prevedere l'assegnazione di una dimora diversa da quella originaria. Vero è che, in presenza di figli, l'accordo è sottoposto al controllo del giudice che deve verificare che esso non arrechi pregiudizio ai figli. Del pari indubbio che i coniugi possono anche - nell'ambito della loro autonomia - decidere di individuare in un'altra dimora (diversa dalla casa domestica) il luogo dove permettere ai figli di continuare a vivere fino al raggiungimento della autosufficienza economica. La casa familiare si fa così strumento di contribuzione al mantenimento,in un

\footnotetext{
${ }^{52}$ Cass., 20 gennaio 2006, n. 1198, in Giur.it., 2006, p. 1595.

${ }^{53}$ Da ultimo, Cass. civ., sez. I, 15 gennaio 2018, (ud. 30/11/2017, dep.15/01/2018), n. 772 in Dejure on line.

${ }^{54}$ Cass., 8 giugno 2012, n. 9371 in Dejure online; Cass. civ., 16 maggio 2013 n. 11981 secondo cui "Il provvedimento di revoca dell'assegnazione della casa coniugale al genitore affidatario del figlio minore è giustificato dal venire meno dell'interesse prioritario del figlio alla continuità ambientale in ragione della lunga permanenza del minore e della madre nella casa dei nonni materni".

${ }^{55}$ Trib. Roma, 7 dicembre 2017 n. 22999 in Dejure online. Per i giudici "La norma di cui all'art 337 sexies c.c., consente di derogare all'ordinario regime privatistico relativo alla proprietà ed al possesso della casa coniugale nell'esclusivo interesse del figlio minore a conservare l'habitat domestico ove ha sempre vissuto". Nel senso di non accettare una eccessiva compromissione del diritto di proprietà, E. QUADRI, L'attribuzione della casa familiare in sede di separazione e divorzio, in Fam.e dir., 1995, p. 283.

${ }^{56}$ Corte cost., 21 ottobre 2005, n. 394, in Foro it., 2007, I, c. 1083 ss.

${ }^{57}$ Introdotto dall'art. 55, dlgs. 28 dicembre 2013, n. 154. Ex multis, Cass., 19 giugno 2013, n. 18440, in Dejure online. Su questi aspetti, G. CARAPEZZA FIGLIA, Assegnazione della casa familiare ed interessi in conflitto, in $L a$ casa familiare nelle esperienze giuridiche. Quaderni di Diritto delle Successioni e della Famiglia, 4, Napoli, 2016, p. 98 ss.

${ }^{58}$ Poi convertito in 1.10 novembre 2014, n. 162.
}

Revista de Direito Brasileira | Florianópolis, SC | v. 23 | n. 9 | p. 389-409 | Mai./Ago. 2019 
contesto nel quale sono le parti a definire la soluzione che meglio le rappresenta. E, tuttavia, se le parti possono scegliere una "casa familiare" diversa da quella domestica, non si vede perché una tale misura non possa essere adottata (ove lo ritenga opportuno) dal giudice ${ }^{59}$. D'altro canto, nel senso dell'adozione di soluzioni più flessibili muove sia l'esperienza francese che quella spagnola.

In Francia, la preferenza per la residenza alternata, quale metodo pratico più adatto ad un esercizio comune e condiviso dell'autorità genitoriale, è evidente nella disposizione secondo cui essa può essere stabilita dal giudice (JAF) anche in caso di disaccordo dei genitori, ovvero su domanda di uno solo dei due.

In simili circostanze, il giudice si può avvalere della facoltà di ordinare la residenza alternata a titolo meramente provvisorio, ovvero fissando un periodo di prova allo scadere del quale, fatte le necessarie verifiche, potrà decidere se, nell'interesse esclusivo del minore, sia o meno opportuno fissarla durevolmente (art. 373-2-9 Code civil).

Ciò perché è piuttosto evidente che, in una situazione di crisi della famiglia, un simile assetto della vita dei minori può funzionare, senza aggravare i dissidi, solo laddove vi sia la seria volontà dei genitori a collaborare su tutti i piani e a distribuirsi equamente il tempo da trascorrere con i figli. Vero è che la loi 305-2002 non regolamenta né le conseguenze patrimoniali né le modalità attraverso le quali è possibile organizzare in concreto la divisione della vita del minore in due case diverse. Del pari indubbio chela lacuna finisce con il rappresentare una vera e propria opportunità per assicurare un migliore funzionamento dello schema di residenza alternata, permettendo al giudice di adattare le soluzioni alle diverse situazioni presentate in giudizio.

Del resto, la soluzione della residenza alternata appare conforme ad un altro principio fondamentale della normativa del 2002 che è quello di favorire, anche attraverso la promozione delle tecniche di mediazione familiare, l'accordo tra i genitori. È confermata, infatti, la possibilità di sottoporre all'omologazione del JAF una convenzione attraverso la quale vengano disciplinate sia le modalità di esercizio congiunto della potestà che gli aspetti patrimoniali relativi al mantenimento della prole (art. 373-2-7 Code civil) ${ }^{60}$.

Anche in Spagna, l'assegnazione della casa familiare presenta indubbie connessioni con il regime dell'affidamento. Alla previsione di cui all'art. 96.1 CC,a norma del quale l'assegnazione della viviendaè compiuta a vantaggio di quello dei coniugi che hanno la custodia dei figli, si affianca un dettato normativo che ha ammesso la custodia compartida, allorchè a chiederlo siano entrambi i coniugi nel convenio regulador ${ }^{61}$. La ricerca di criteri alternativi di attribuzione della vivienda si è accentuato nel momento in cui la giurisprudenza - ragionando sull'art. $92.8 \mathrm{CC}$ - ha cominciato a non considerare più eccezionali i provvedimenti di custodia compartida ${ }^{62}$. Si è cosi sviluppata, per quanto riguarda la questione dell'assegnazione della casa familiare, una giurisprudenza"alternativa"che, quando il provvedimento non è contrario all'interesse del minore, ritiene soddisfatto il suo interesse anche disponendo il godimento di un'altra abitazione della coppia.

La messa in discussione del principio secondo il qualeinteresse del minore è quello di rimanere nella stessa casa dove si è svolta la vita familiare, si fonda su una interpretazionedell'art. $96 \mathrm{CC}$ che ricostruisce l'attribuzione della vivienda in chiave di

\footnotetext{
${ }^{59}$ G. CARAPEZZA FIGLIA, Assegnazione della casa familiare ed interessi in conflitto, cit., p. 109. Più in generale sulla tendenza alla privatizzazione delle relazioni familiari, M. SESTA, Privato e pubblico nei progetti di legge in materia familiare, in AA.VV., Studi in onore di P. Rescigno, II, 1; Milano, 1998, p. 829 ss.

${ }^{60}$ In Cass.civ., 25 aprile 2007, in Rev.trim.dr.civ., 2007, p. 560 i giudici osservano come la collocazione esclusiva presso uno dei genitori possa essere fonte di tensioni, concludendo nel senso della preferibilità della "residence en alternance"; Y. BUFFELAN-LANORE, Domicile, demeure et logement familial, in Rép.Civ.Dalloz, 2015 , § 21 ss.

${ }^{61}$ Cfr., la modifica dell'art. 92 CC a seguito della Ley 8 luglio 2005. In giurisprudenza, fra le tante STS 19 abril 2012 /RAJ 2012, 5090 e STS 29 abril 2013 (RAJ 2013, 3269).

62 A.I. BERROCAL LANZAROT, El intéressupeiror del menor y la atribución de la guarda y custodia, en rev. Crítica de Derecho Immobiliario, 2014, n.746, p. 3284 ss.
}

Revista de Direito Brasileira | Florianópolis, SC | v. 23 | n. 9 | p. 389-409 | Mai./Ago. 2019 
prestazione alimentare. Il fatto di guardare all'attribuzione della casa familiare come ad un modo di contribuire al mantenimento dei figli permettecosì ai giudici spagnoli di evitare l'assegnazione ogni qual volta i figli possano contare su altra dimora ${ }^{63}$ o quando esista una soluzione alternativa che consenta di evitare "las facultades de disposición del derecho de la propriedad que ostenta el recurrente sobre la vivienda que actualmente ocupan el menor y su madre"64.

La tutela degli interessi del minore esce così da quella dimensione astratta e stereotipata cui la costringono i giudici italiani preoccupati più di offrirne una ricostruzione che di procedere ad una verifica dei fatti attraverso cui dare concretezza al principio.

\section{A PROPOSITO DI FALSI MITI: IL MINORE PROTAGONISTA E LA INFORMED DOCTRINE FORMULA}

Nell'intreccio complesso della esperienza umana il sein si sovrappone al sollen e sul consenso si scarica la fragilità della persona; una fragilità che evidenzia il modo in cui "le polimorfe reazioni psichiche alla malattia" temperino schemi e modelli comportamentali e impediscano ricostruzioni rigide destinate a sfociare in quella sequenza paternalismo, autonomia, burocrazia parsimoniosa dal sapore vagamente ricostruttivo. Certo l'obbligo di informare accuratamente il paziente dell'irreversibilità della propria condizione di salute ha molto a che vedere con le dinamiche di una razionalità che permette la programmazione delle vicende future. Ma l'essere razionale sembra esistere più in teoria che nella pratica, come dimostrano le neuroscienze cognitive e come si ricava dagli sforzi compiuti per lo più da un diritto di rango pretorio che si preoccupa di precisare le condizioni di validità del consenso nonché di circondarlo di quelle garanzie e condizioni che lo rendano, appunto, libero e consapevole.

Sta di fatto che quando condizioni patologiche o legate all'età rendono dubbia la consapevolezza della decisione, un legislatore, così dichiarando la propria fedeltà al principio di autodeterminazione, è costretto a "procedimentalizzare" il processo decisionale chiamando in causa un terzo: personne de confiance, representante legal, amministratore di sostegno. Ma, quando lo fa, schermandosi dietro la necessità di protezione della persona vulnerabile, come nel caso del mandat de protecion future $e^{65}$ non si accorge che la condizione di impossibilità a decidere (presupposto della protezione) è più difficile da determinare di quanto sembri, con la conseguenza di rendere labile il confine tra autonomia e sottomissione a un'autorità esterna, portando acqua al mulino del ragionamento foucaultiano. E soprattutto con il rischiodi spostare, nell'affannosa ricerca della volontà, l'asse della riflessione sul giudice (che ancora meno del medico è legittimato ad assumere decisioni) determinandosi, in nome proprio della vulnerabilità della persona, una giudizializzazione della sua tutela. Il paradosso risiede nel fatto che quella dimensione paternalistica da cui ci si era faticosamente allontanati risorge o, piuttosto, cambia pelle, favorita proprio da una esasperata ricerca della volontà, definitivamente mostrando la fragilità ontologica della informed doctrine formula e dando ragione a chi suggerisce di liberarsi da falsi miti ${ }^{66}$.

Né le cose cambiano rispetto al minore. A parte declamatorie formule che rinviano alla necessità di ascolto della sua volontà e capacità di discernimento, informazione sanitaria e decisione chiamano in causa in primis i genitori e, in ipotesi di conflitto, i giudici. In questi casi

\footnotetext{
${ }^{63}$ STS 29 marzo 2011 (RAJ 2011, 3021).

${ }^{64}$ STS 16 jenero 2015 (JUR 2015, 3073).

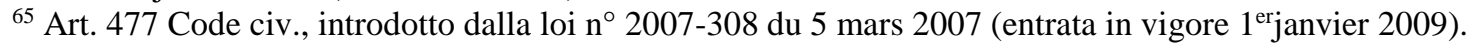

${ }^{66}$ R. M. VEATCH, Abandoning informed consent, Hastings Center Report, 1995, 25(2), pp. 5-12. Per un diversoapproccio in polemica con Veatch e chetenta di fornireargomentianchefilosofici a fondamento del consenso, B. Cox White e J. Zimbelman, Abandoning Informed Consent: An Idea Whose Time Has Not Yet Come, in Journal of Medicine and Philosophy, 1998, 23, (5), pp. 477-499.
}

Revista de Direito Brasileira | Florianópolis, SC | v. 23 | n. 9 | p. 389-409 | Mai./Ago. 2019 
la valutazione del best interest diventa lo scudo sotto il quale riassorbire tutte quelle decisioni che potrebbero essere contrarie alla salute del paziente. Uno scudo che, a seconda dei casi, viene utilizzato ora dai genitori ora dal giudice, come pure sembrerebbe confermare la vicenda di David Glass $^{67}$. Anche quando "l'affrancamento dallo stereotipo della capacità di agire e la fissazione di un'età differente" sembrano risolvere la questione del consenso del minore - come accade in Spagna e in Inghilterra - è emblematico che ciò si verifichi solo per decisioni declinate in positivo. Né, a dispetto della diversità di approccio seguito nei diversi Stati americani, la mature minor doctrine fa eccezione alla regola, visto che, per un verso, si tratta di regola priva di generale applicazione e, per l'altro, pone il problema di come valutare l'effettiva capacità dell'adolescente in quanto " The legal principles judges use to decide cases about children are often vague $[\ldots .$.$] research concerning child development suggests that concepts like "knowing,$ intelligent, and voluntary"-while somewhat immutable when applied to the adult reasoning process-is fluid prior to adulthood" 68 . Il che, in rude sintesi, induce a ritenereche "the current case law and statutes do not give any clear guidance (to resolve) the problem. They tell you the principles to apply but not how these principles apply to your case" ${ }^{69}$. Ma v'èdell'altro.

Se è veroche, conformeallatradizione di common law, in InghilterrailGillick competence testfissa un principio ben precisochesiritrovascolpitonelle parole di Lord Scarman per il quale "as a matter of law, the parental right to determine whether or not their minor child below the age of 16 will have medical treatment terminates, if and when, the child achieves sufficient understanding and intelligence to understand fully what is proposed"; è ancheveroche la difficoltà di cogliere la maturitàdell'adolescente ha indotto, più di recenteigiudici, alla luce dellaprevisione di cui alla $\S 1(2)$ MCA 2005, a leggere "across to, and borrow from, the relevant concepts and language of the Mental Capacity Act 2005" (para 15)""70. Di qui, allora, a conferma della difficoltà di valutare la consapevolezza del consenso, il rifiuto di una visione legata solo all'età e, piuttosto, l'esigenza di approdare ad una valutazione della capacità nel contesto specifico delle circostanze, poiché si osserva che "removing the specific factual context from some decisions leaves nothing for the evaluation of capacity to bite upon"71.

Nella babele delle riflessioni che circondano il dibattito sul consenso, il punto è proprio quello di verificare se, quando l'informazione è stata veicolata il minore fosse nelle condizioni di capirla, (anche alla luce delle conseguenze che produce), metabolizzarla, valutarla e comunicare la propria volont ${ }^{72}$ à. Nell'ordinamento italiano, il caso dei Testimoni di Geova è indicativo di queste difficoltà. Il timore del medico di non riuscire a cogliere correttamente la volontà del minore in situazioni di pericolo per la sua vita sembra qui superare lo schermo della rilevanza da assegnare alle convinzioni religiose, sfociando in quel "sentimentalismo" del medico italiano capace di far i suoi maggiori danni. D’altro canto, proprio quella dei Testimoni di Geova è vicenda per più versi di complessa soluzione, specie quando in discussione è la necessità di bilanciare il diritto alla libertà religiosa con le esigenze di tutela del minore ${ }^{73}$, in presenza di pratiche religiose che fanno emergere dei rischi. In proposito la giurisprudenza europea (Palau-

\footnotetext{
${ }^{67}$ Glass v UK (Application No 61827/00) [2004] 1 FCR 553.

${ }^{68}$ W.J. MLYENIC, A Judge Ethical's Dilemma: Assessing a Child Capacity to Choose, in Fordham l. Rev., 1996, (64), $1873,1874$.

${ }^{69}$ N. MORRIS, The Brothel Boy and other parables of the law, 1998, p. 45.

${ }^{70}$ Re S (Child as parent: Adoption: Consent) [2017] EWHC 2729 (Fam).

${ }^{71}$ City of York Council v C [2013] EWCA Civ 478; [2014] Fam 10 at [35]

72 Sull'argomento e così per una più approfondita analisi della citata evoluzione sia consentito il rinvio a $\mathrm{P}$. STANZIONE, Capacità e minore età nella problematica della persona umana, Camerino-Napoli, 1975, passim; ID., Diritti fondamentali dei minori e potestà dei genitori, in Rass. dir. civ., 1980, p. 446 ss.; ID., Scelte esistenziali e autonomia del minore, in Rass. dir. civ., 1983, 11, p. 1145 ss. ; ID., Capacità (diritto privato), in Enc. giur. Treccani, Roma, V, 1988, 1 ss.; ID., Interesse del minore e "statuto" dei suoi diritti, in Fam. e dir., 1994, p. 351 ss.

${ }^{73}$ CEDH, 16 déc. 2003, Palau-Martinez c. France, req. n 64927/01, D. 2004. 1261; CEDH, 23 giugno 1993, Hoffman c. Austria, req. ${ }^{\circ} 12875 / 87$.
}

Revista de Direito Brasileira | Florianópolis, SC | v. 23 | n. 9 | p. 389-409 | Mai./Ago. 2019 
Martinez v France ma anche Hoffman c. Austria) lungi dal fare applicazione di un astratto criterio di valutazione dell'interesse del minore mostra di sviluppare un approccio equilibrato che rifiuta una valutazione in astratto del suo interesse; valutazione che si trasformerebbe in una "directive générale et impersonnelle" 74 soprattutto quando in discussione è il diritto del minore ad intrattenere rapporti con il padre e con la madre testimone di Geova. Ancora una volta, in discussione non è il principio della tutela del minore o il riconoscimento astratto della sua autodeterminazione ma la valutazione delle circostanze di fattonella quali la autodeterminazione si esplica. Sì che, sebbene 1'art. 12 della Convenzione di New York affermi il diritto del minore ad essere informato e poter esprimere le proprie decisioni e la Convenzione di Strasburgo ribadisca il diritto del minore di essere consultato ed esprimere la propria opinione (art. 3) non è agevole immaginare di "individuare" nel minore stesso il miglior difensore dei propri interessi.

Che fare allora?

Qualunque sia la prospettiva che si assume, la presa in carico del tema dell'autodeterminazione del minore e della sua capacità di discernimento sembra dipendere molto dal contesto culturale, dalle tecniche elaborate nei diversi contesti ordinamentali e dalle situazioni concrete in cui ci si trova ad operare. Lo si voglia o no, nel caso delle scelte relative alla salute, si è in presenza di situazioni di incertezza, cui neppure un attento legislatore, puntigliosamente impegnato a definire le coordinate di libera manifestazione del consenso può porre rimedio. La vicenda del minore è un esempio della inadeguatezza del legislatore a governare adeguatamente le questioni bioetiche e di autodeterminazione. Chiedersi quale sia lo spazio riservato all'autodeterminazione in salute significa allora chiedersi quali siano, in campo sanitario, le coordinate di emersione delle scelte personali, privilegiando una visione che tenga conto dell'irriducibilità dell'esperienza umana e leghi, pertanto, la salute ad una concezione personale della cura, così come della malattia. Ma significa anche verificare la idoneità delle corti in fondo meglio equipaggiate del legislatore perchè "concerned with the flesh and blood of an actual case" a risolvere le complesse questioni che solleva l'autodeterminazione in generale e quella del minore in particolare.

\section{CONCLUSIONE}

Scopo della presente indagine era quello di verificare il livello di protezione offerto al minore a prescindere dal suo status familiare. Il rispetto della sua dignità e dei suoi diritti è funzionale allo sviluppo della personalità del minore che è persona "in formazione".

La verifica della ipotesi di partenza è stata raggiunta grazie all'analisi della legislazione straniera e italiana, nonché delle posizioni assunte, sul punto, dalla dottrina e dalla giurisprudenza.

Filiazione, identità e interesse del minore sono aspetti strettamente correlati. Il dirittodel figlio adottivo, o nato da tecniche di fecondazione assistita, a conoscere le proprie origini è un diritto intrinsecamente legato alla personalità; così come strettamente legato alla personalità è il diritto del minore a "mantenere relazioni significative" con i membri della famiglia diversi dai genitori (ad esempio i nonni, che pure fanno parte degli affetti che circondano il minore).

L'interesse del minore alla stabilità della relazione affettiva supera, dunque, esclusioni e normative irrazionali, aprendo anche alla possibilità di ammettere l'adozione del figlio del partner nel caso di una coppia omosessuale (cd. step child adoption). Il diritto di conoscere le origini si afferma, invece, come un diritto fondamentale della persona; diritto che è autonomo rispetto all'identità e deriva la sua ragion d'essere dalla necessità di conoscere le radici della

${ }^{74}$ CEDH, 16 déc. 2003, Palau-Martinez c. France, cit., § 37. Revista de Direito Brasileira | Florianópolis, SC | v. 23 | n. 9 | p. 389-409 | Mai./Ago. 2019 
propria esistenza e, come nel caso dell'adozione, di un'esperienzache ha generato un contesto relazionale.

Su queste tematiche la giurisprudenza non offre risposte univoche. Ne consegue che è compito dela dottrina rilevare incoerenze e contraddizioni, nella prospettiva di una più adeguata tutela del minore.

\section{BIBLIOGRAFIA}

G. AUTORINO STANZIONE, Introduzione, in Il diritto di famiglia nella dottrina e nella giurisprudenza. Trattato teorico-pratico, Torino, $2^{\mathrm{a}}$ ed., 2011, I, p. XV ss.

M. SESTA, Diritto di famiglia, Padova, 2005, 1 ss.

A. FALZEA, Nel cinquantenario della convenzione europea sui diritti dell'uomo, in Riv.dir.civ., 2000, p. 696.

J. CARBONNIER, Les notions à contenu variable dans le droit français de la famille, inC. Perelman et R.

Vander Elst (dir.), Les notions à contenu variable en droit, éd. Bruxelles. E. Bruylant, 1984, p. 99. J. GOLDSTEIN, FREUD, ANNA e SOLNIT, ALBERT, In The Best Interests Of The Child, New York Free Press 1986, passim. Per una attenta e completa definizione dell'interesse del minore si rinvia a P. STANZIONE, Interessi del minore $e<\langle$ statuto $\rangle>$ dei suoi diritti fondamentali, in Fam. e dir., 1994, p. 351 ss.;

G. AUTORINO, Diritto di famiglia, Torino, 2003, p. 195. In proposito, altresì, G. CASABURI, Nota a Cass, civ. 20 gennaio 2006, n. 1202, in Foro it., 2006, 5, c. 1410 ss. nonché F. RUSCELLO, La tutela dei figli nel nuovo 〈〈affidamento condiviso >>, in Familia, 2006, passim; A. SCALISI, Famiglia e diritti del minore, in Fam. pers. e succ., 2006, 10, p. 817 ss.; C.

M. BIANCA, La nuova disciplina in materia di separazione dei genitori e affidamento condiviso: prime riflessioni, in Dir. fam. e pers., 2006, XXXV, 2, p. 678; G. SALITO, $L$ 'affidamento condiviso dei figli nella crisi della famiglia, in Diritto di Famiglia. Trattato teorico-pratico, dir. da G. Autorino Stanzione, Torino, 2007, p. 231 ss. Sul punto sia consentito, altresì, il rinvio a V. ZAMBRANO, Interesse del minore ed affidamento coniugato. Esperienze europee a confronto, in Dir. fam. e pers., 2000, 3-4, p. 1385.

G. ZACCARIA, Precomprensione, princìi e diritti nel pensiero di Josef Esser. Un confronto con Ronald Dworkin, in "Ragion pratica", 1998, 6, p. 145; G. PINO, Principi e argomentazione giuridica, in "Ars Interpretandi. Annuario di ermeneutica giuridica", 2009, p. 144.

H. FULCHIRON, Existe-t-il un modèle familial européen?,Defrénois 2005, p. 1461.

V. SCALISI, La "famiglia" e le "famiglie" (il diritto di famiglia a dieci anni dalla riforma), in Scritti catanzaresi in onore di Angelo Falzea, Napoli, 1987, p.431 ss.; ID., "Famiglia” e "famiglie" in Europa, relazione svolta al convegno

Persona e comunità familiare, 1982-2012, in www.comparazionedirittocivile.it; V. CARBONE, Crisi della famiglia e principio di solidarietà, relazione svolta al convegno Persona e comunità familiare, 1982-2012, C. MAZZÙ, Nuovi modellidi status nel diritto di famiglia,

F.D. BUSNELLI, La famiglia e l'arcipelago familiare, in Riv. dir. civ., 2002, I, p. 509 ss. 
D. BORILLO, La vérité biologique contre l'homoparentalité: le statut du beauparentou le «PaCS de la filiation», in Droit et société 2009/2, n. 72, p. 259-271.

MAUGER-VIELPEAU, La volonté et la filiation au regard du droit nouveau. (A propos de l'Ordonnance n. 2005-759 du 4julliet 2005 portant réforme de la filiation), in Pet. Aff., 27 juin 2007, 128, p. 3 ss.

F.D. BUSNELLI, Sui criteri di determinazione della disciplina normativa della famiglia di fatto, in Famiglia di fatto. Atti del convegno di Pontremoli, Pontremoli, 1977, p. 133;

F. SANTORO-PASSARELLI, Parentela naturale, famiglia e successione, in Riv. trim. dir. proc. civ., 1981, p. 27

R. BIN, La famiglia: alla radice di unossimoro, in Studium juris, 2000, p. 1068. Le "nuove famiglie" e la parificazione degli status di filiazione ad opera della L. 219/2012. Atti dei Convegni di Milano, 7 marzo M.G. STANZIONE, Il diritto alla genitorialità e alle relazioni familiari, in www.comparazionedirittocivile.it, p. 5.

G. FERRANDO, Stato unico di figlio e varietà dei modelli familiari, in Fam.dir., 2015, p. 952. Osserva OLG Stuttgart 24 agosto 2016 in Aktenzeichen 17 UF 40/16. Più in generale, sulla necessità di approdare ad una valutazione concreta di ciò che corrisponde all'interesse del minore, anche tenedo conto delle sue aspirazioni, cfr., BVerfG, FamRZ, 2008, s. 1737 ff. E, in dottrina, Staudinger/Coester (2015) § 1666 Rn. 66.

J. LONG, Il diritto italiano della famiglia alla prova delle fonti internazionali, Milano, 2006, 68 ss. In GU Serie Generale n.118 del 21 maggio 2016.

E. ANTONIO EMILIOZZI, L'adozione da parte di partners di unioni civili, in Riv.trim.dir.proc.civ., fasc.2, 1, 2018, pag. 799; V. CARBONE, Riconosciute le unionicivili tra persone dello stesso sesso e le convivenze di fatto, in Fam. e dir., 2016, pp. 856-857; G.

FERRANDO, La disciplina dell'atto. Gli effetti: diritti e doveri, in Fam. e dir., 2016, p. 894; Trib.min. Roma, 22 ottobre 2015, in Foro it., 2016, I, c. 339 ss.

P. MOROZZO DELLA ROCCA, Sull'adozione da parte degli affidatari dopo la L. n. 173/2015, in Fam. e dir., 2017, p. 602 ss.

V. ZENO ZENCOVICH, voce Identità personale, in Digesto delle discipline privatistiche, IX, Torino, 1993, p. 294.

F. C. SESSAREGO, Derecho a la identidad personal, Buenos Aires, 2012, p. 15 ss.; M. HERRERA, E. LAMM, Sub art. 564, in Tratado de derecho de familia, Según el Código civil y commercial de 2014, t. II, artt. 509-593, Buenos Aires, 2014, p. 558 ss.

J.G.FICHTE, La missione del dotto, La Nuova Italia, Firenze 1939 e 1973, passim.

G. CORNU, Droit civil. La famille, Paris, Montchrestien, 9e éd., 2006; D. BORILLO, La vérité biologique

contre l'homoparentalité: le statut du beau-parento u le "PACS de la filiation", in Droit et sociétés, vol. 2, pp. 259-271.

L. 28 marzo 2001, n. 149. 
J. LEDESMA DEL BUSTO, Mediación familiar en búsqueda de orígens. El encuentro con mi espejo biológico, Madrid, 2012, passim.

LA. LAMARCA I MARQUE, Autonomía privada e intervención pública en las acciones de filiación. La reforma del BGB, in http://www.indret.com/pdf/561_es.pdf.

G. CARAPEZZA FIGLIA, Assegnazione della casa familiare ed interessi in conflitto, cit., p. 109. Più in generale sulla tendenza alla privatizzazione delle relazioni familiari, M. SESTA, Privato e pubblico nei progetti di legge in materia familiare, in AA.VV., Studi in onore di P. Rescigno, II, 1; Milano, 1998, p. 829 ss.

A.I. BERROCAL LANZAROT, El intéressupeiror del menor y la atribución de la guarda y custodia, en rev.Crítica de Derecho Immobiliario, 2014, n.746, p. 3284 ss.

R. M. VeATCH, Abandoning informed consent, Hastings Center Report, 1995, 25(2), pp. 5-12.

Per un diversoapproccio in polemica con Veatch e chetenta di fornireargomentianchefilosofici a fondamento del consenso, B. Cox White e J. Zimbelman, Abandoning Informed Consent: An Idea Whose Time Has Not W.J. MLYENIC, A Judge Ethical's Dilemma: Assessing a Child Capacity to Choose, in Fordham l. Rev., 1996, (64), 1873, 1874.

N. MORRIS, The Brothel Boy and other parables of the law, 1998, p. 45.

CEDH, 16 déc. 2003, Palau-Martinez c. France, req. n 64927/01, D. 2004. 1261; CEDH, 23 giugno 1993, Hoffman c. Austria, req. n 12875/87. 